\title{
Analysis of parent of origin specific DNA methylation at SNRPN and PW71 in tissues: implication for prenatal diagnosis
}

Diagnostic

Development Branch, National Center for

Human Genome

Research, NIH,

Bethesda, Maryland,

USA

T Kubota

S L Christian

D H Ledbetter

Department of

Obstetrics and

Gynecology,

Georgetown University

Medical Center,

Washington DC, USA

S Aradhya

M Macha

A C M Smith

Children's Hospital of Eastern Ontario,

Canada

L C Surh

Sinai Hospital of

Baltimore, Molecular

Pathology-Clinical and

Molecular

Cytogenetics,

Maryland, USA

J Satish

University of Chicago, Illinois, USA

M S Verp

Prenatal Diagnostic

Center,

Massachusetts, USA

$\mathrm{H}$ L Nee

A Johnson

Correspondence to:

Dr Ledbetter, Center for

Medical Genetics, MC 2050,

The University of Chicago,

5841 South Maryland

Avenue, Chicago, IL 60637-

1470 , USA.

Received 15 May 1996

Revised version accepted for publication 1 July 1996

\begin{abstract}
Takeo Kubota, Swaroop Aradhya, Michelle Macha, Ann C M Smith, Linda C Surh, Jayavanthi Satish, Marion S Verp, Holly L Nee, Anthony Johnson, Susan L Christian, David H Ledbetter
\end{abstract}

\begin{abstract}
Prader-Willi syndrome (PWS) and Angelman syndrome (AS) are distinct developmental disorders caused by absence of paternal or maternal contributions of the chromosome region 15q11-q13, resulting from deletions, uniparental disomy (UPD), or rare imprinting mutations. Molecular cytogenetic diagnosis is currently performed using a combination of fluorescence in situ hybridisation (FISH), DNA polymorphism analysis, and DNA methylation analysis. Only methylation analysis will detect all three categories of PWS abnormalities, but its reliability in tissues other than peripheral blood has not been examined extensively. Therefore, we examined the methylation status at the CpG island of the small nuclear ribonucleoprotein associated polypeptide $\mathbf{N}$ (SNRPN) gene and at the PW71 locus using normal and abnormal lymphoblast (LB) cell lines $(n=48)$, amniotic fluid (AF) cell cultures $(n=25)$, cultured chorionic villus samples (CVS, $n=17$ ), and fetal tissues $(n=18)$ by Southern blot analysis with methylation sensitive enzymes. Of these samples, 20 LB cell lines, three AF cultures, one CVS, and 15 fetal tissues had been previously diagnosed as having deletions or UPD by other molecular methods. Methylation status at SNRPN showed consistent results when compared with FISH or DNA polymorphism analysis using all cell types tested. However, the methylation pattern for PW71 was inconsistent when compared with other tests and should therefore not be used on tissues other than peripheral blood. We conclude that SNRPN, but not PW71, methylation analysis may be useful for diagnosis of PWS/AS on LB cell lines, cultured amniotic fluid, or chorionic villus samples and will allow, for the first time, prenatal diagnosis for families known to carry imprinting centre defects.

(f Med Genet 1996;33:1011-1014)
\end{abstract}

Key words: SNRPN methylation; PW71 methylation; Prader-Willi/Angelman syndromes; prenatal diagnosis.

Prader-Willi syndrome (PWS) is usually caused by a paternal deletion or maternal uniparental disomy (UPD) of the chromosome region 15q11-q13. Angelman syndrome (AS) is caused by a maternal deletion or paternal UPD of the same region on chromosome 15 in the majority of cases. A third mechanism of PWS/ AS has recently been proposed involving a putative imprinting centre located upstream of the SNRPN gene through identification of small deletions in several cases with sporadic or familial PWS or AS. ${ }^{12}$ Molecular cytogenetic diagnosis for PWS/AS has been performed using fluorescence in situ hybridisation (FISH) to identify deletions ${ }^{34}$ and DNA polymorphism (microsatellite) analysis ${ }^{56}$ to identify either deletions or UPD of chromosome 15. However, neither of these methods will detect the rare category of imprinting mutations, which are sometimes found in familial cases with substantial recurrence risks.

DNA methylation analysis at the PW71 locus or at the $\mathrm{CpG}$ island (exon $\alpha$ ) of the small nuclear ribonucleoprotein associated polypeptide $\mathrm{N}$ gene (SNRPN) can detect all three major classes of genetic defects associated with PWS/AS (deletions, UPD, or imprinting mutation) using peripheral blood samples, although these analyses will not discriminate between them. ${ }^{78}$ However, PW71 methylation analysis does not provide accurate results for PWS/AS diagnosis using cultured fibroblast (FB) cells ${ }^{9}$ or chorionic villus samples (CVS). ${ }^{9-11}$

To determine whether tissues other than peripheral blood are suitable sources for SNRPN or PW71 methylation analysis, we examined methylation at the above two loci in lymphoblast (LB) cell lines, amniotic fluid (AF) cells, chorionic villus samples (CVS), and fetal tissues on a series of normal controls and PWS/ AS patients with known deletion/UPD status.

\section{Materials and methods}

PATIENTS

Methylation analyses were performed on genomic DNA extracted from a total of 90 cell cultures including $48 \mathrm{LB}$ cell lines, $22 \mathrm{AF}$ samples, and $17 \mathrm{CVS}$. Of $48 \mathrm{LB}$ samples tested, a paternal deletion (PWS) had been confirmed in 10 samples, a maternal deletion (AS) had been confirmed in seven samples by FISH and DNA polymorphism analyses, and maternal UPD15 (PWS) had been confirmed in three samples by DNA polymorphism analysis. Seven LB cell lines with paternal deletions and three LB cell lines with maternal deletions were obtained from the NIGMS Human Genetic 
Table 1 Summary of methylation results for SNRPN and PW71

\begin{tabular}{lcllllll}
\hline & Normal & Pat deletion & Mat deletion & MatUPD15 & Total & $\%$ Discrepancy \\
\hline SNRPN & & & & & & & \\
LB & 28 & $(0)$ & $10(0)$ & $7(0)$ & $3(0)$ & $48(0)$ & 0 \\
AF & $22(0)$ & 0 & 0 & $3(0)$ & $25(0)$ & 0 \\
CVS & $16(0)$ & 0 & 0 & $1(0)$ & $17(0)$ & 0 \\
PW71 & & & & & & & \\
LB & $28(3)$ & $10(3)$ & $7(2)$ & $3(0)$ & $48(8)$ & 17 \\
AF & $22(8)$ & 0 & 0 & $3(0)$ & $25(8)$ & 32 \\
CVS & $16(16)$ & 0 & 0 & $1(1)$ & $17(17)$ & 100 \\
\hline
\end{tabular}

Normal $=$ normal subjects or patients with biparental chromosomes 15

$(\mathrm{)}=$ number of samples which showed discrepancy between methylation analysis and othe molecular cytogenetic analyses.

Mutant Cell Repository (Camden, NJ). Other LB cell lines were established from peripheral blood of patients submitted to our laboratory for diagnosis of PWS/AS or other syndromes.

Of $25 \mathrm{AF}$ samples tested, maternal UPD15 had been confirmed in three samples. Of 17 CVS analysed, maternal UPD15 had been confirmed in one sample. Most AF and one CVS samples were sent to our laboratory for prospective prenatal diagnosis for UPD using microsatellite analysis. One AF sample with maternal UPD15 has been previously published. ${ }^{12}$ Most normal CVS were provided from routine prenatal testing (Genetics \& IVF Institute, Fairfax, VA). All other cases with UPD or deletions were confirmed by DNA polymorphism analysis or FISH or both in our laboratory.

Cultured fetal tissues were also analysed in one control case which had been referred for mosaic trisomy 16 in CVS, and in two cases with UPD 15 mat ascertained with either mosaic trisomy 15 in CVS, or a balanced $45, \mathrm{XX}$, $\mathrm{i}(15)$ (q10) in AF. DNA polymorphism analysis confirmed maternal UPD16 in case 1 (unpublished data) and maternal UPD15 in both case $2^{13}$ and in case 3 (unpublished data). Following genetic counselling each family elected to terminate the pregnancy and fetal tissue samples were sent to our laboratory for analysis.

METHYLATION ANALYSIS

DNA was extracted from cultured cells and tissues using standard methods and SNRPN methylation analysis was performed as described previously. ${ }^{8}$ Briefly, genomic DNA was digested with $X b a \mathrm{I}$ and $N o t \mathrm{I}$ and separated on a $1.5 \%$ agarose gel. As an internal control, DNA from a cosmid clone, which contains a NotI site in the multiple cloning site of the vector, was used to confirm complete digestion. Southern transfer was performed onto a Hybond $\mathrm{N}+$ nylon membrane (Amersham Inc, Arlington Heights, IL). As probe, a $0.9 \mathrm{~kb}$ NotI fragment cut from a $4.2 \mathrm{~kb}$ XbaI fragment containing the SNRPN exon $\alpha^{1}$ (DNA probe is available from ATCC/NIH Repository, No 95678, 95679) was radiolabelled and hybridised to Southern blots. Following hybridisation, filters were washed to a final stringency of $0.1 \times \mathrm{SSC} / 0.1 \% \mathrm{SDS}$ at $65^{\circ} \mathrm{C}$. Autoradiography was performed for six to 20 hours.

PW71 methylation analysis was performed as described previously using radiolabelled PW71B probe hybridised to a filter containing genomic DNA digested with HindIII and CfoI. ${ }^{101415}$

\section{Results}

ANALYSIS OF TISSUE SAMPLES

Examples of methylation at the NotI sites in the CpG island of the SNRPN gene for LB
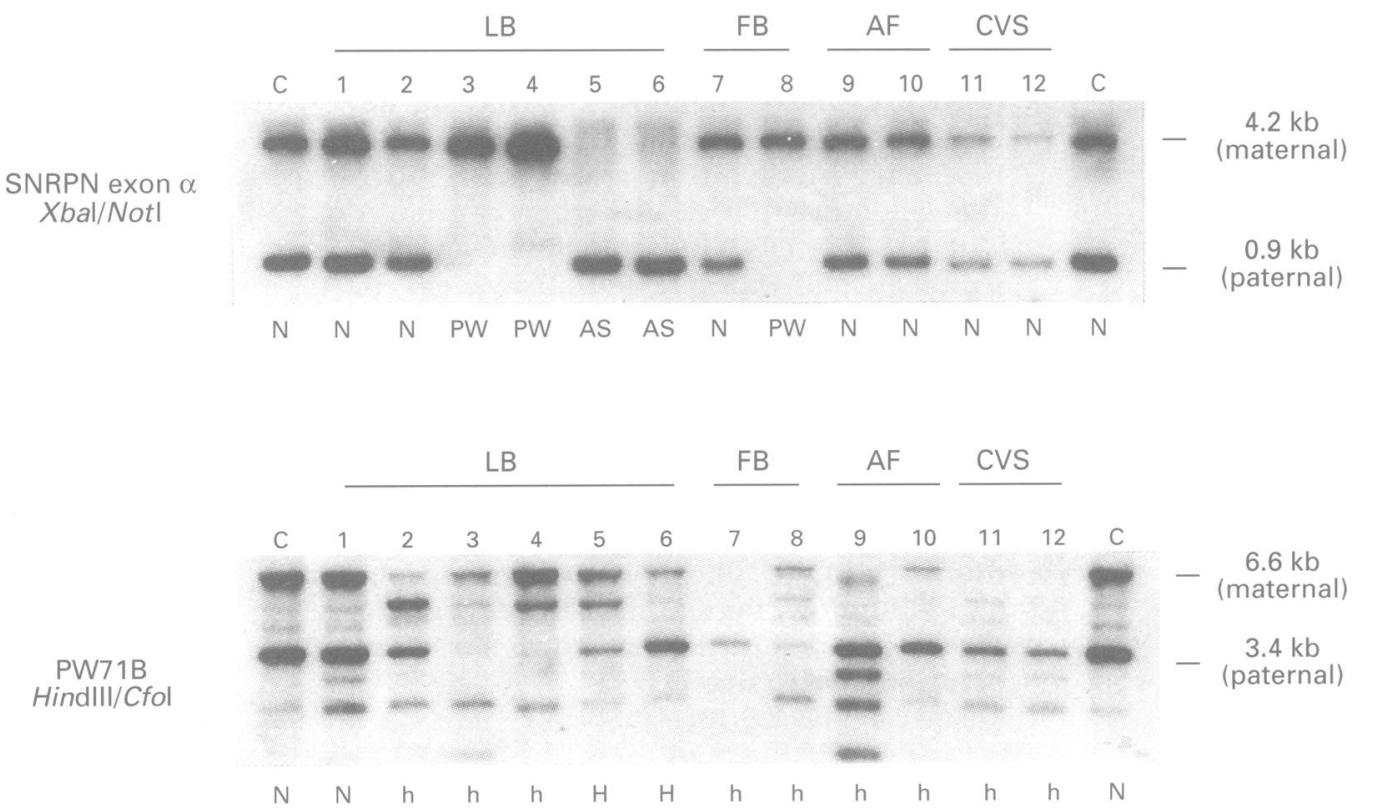

Figure 1 Methylation analysis of cultured tissues for SNRPN (top panel) and PW71 (lower panel) in lymphoblast (LB) cell lines, fibroblast $(F B)$ cell cultures, amniotic fluid $(A F)$ cell cultures, and cultured chorionic villus samples (CVS). For SNRPN, DNA from normal control (C) lymphocyte and normal (N) cell lines (lanes 1, 2, 7, 9-12) shows the expected maternal and paternal bands, while the Prader-Willi (PW) lanes $(3,4,8)$ show only the $4.2 \mathrm{~kb}$ maternal the expected maternal and paternal bands, while the Prader-Willi (PW) lanes $(3,4,8)$ show only the $4.2 \mathrm{~kb}$ mat
band and the Angelman (AS) lanes $(5,6)$ show a single $0.9 \mathrm{~kb}$ paternal band. For PW71B, the control (C) lymphocyte DNA shows the expected maternal and paternal bands. Hypomethylation $(h)$ was observed in both normal and PWS cell lines, while hypermethylation $(H)$ was observed in two AS cell lines (lanes 5, 6). 


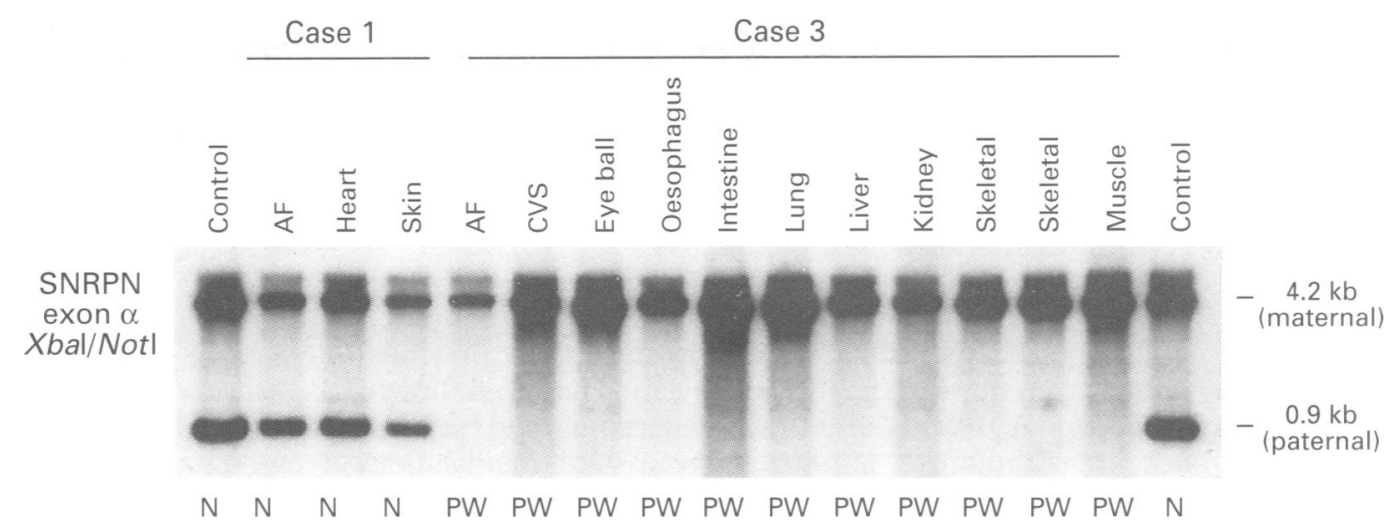

Figure 2 SNRPN methylation analysis of fetal tissues in one case of UPD16, but normal biparental inheritance for chromosome 15 (case 1) and one case of UPD15mat (case 3). Control = blood DNA control, AF=amniotic fluid cells, $F B=$ fibroblast cells, $C V S=$ chorionic villus sample. $N=$ normal pattern, $P W=P W S$ pattern. Note: methylation status of $A F$ is consistent with that of fetal tissues in both cases.

cell lines, AF, and CVS are shown in fig 1. A $4.2 \mathrm{~kb}$ undigested maternal and a $0.9 \mathrm{~kb}$ digested paternal band (normal pattern) were detected in all samples with normal, biparental inheritance. Only a $4.2 \mathrm{~kb}$ maternal band (PWS pattern) was detected in all samples with either a paternal deletion of $15 \mathrm{q} 11-\mathrm{q} 13$ or maternal UPD 15, and only a $0.9 \mathrm{~kb}$ paternal band (AS pattern) was detected in all samples with a maternal deletion of 15q11-q13. In all samples, the SNRPN methylation results were completely consistent with results of FISH and DNA polymorphism analysis (table 1).

Examples of the methylation at the $C f o$ I sites at PW71 on these same samples are also shown in fig 1. However, methylation results were not consistent with previous analyses of DNA polymorphism or FISH in $17 \%$ of LB samples, $32 \%$ of AF samples, and $100 \%$ of CVS (table 1). Additional smaller bands and absent or a weak maternal $6.6 \mathrm{~kb}$ band were seen in LB, fibroblast, $A F$, and CVS samples (fig 1, bottom panel, lanes 2-4,7-12), whereas the same DNA samples showed clear results with the SNRPN probe (fig 1, top panel). The presence of additional smaller bands and absence or weakness of the undigested maternal $6.6 \mathrm{~kb}$ band suggests that the $C f o$ I sites at PW71 are hypomethylated on the maternal chromosome 15 compared with methylation status at the NotI

Table 2 Methylation results in fetal tissues

\begin{tabular}{lllll}
\hline Case & Gestation (wk) & Tissue & SNRPN pattern & PW71 pattern \\
\hline 1 UPD16mat & 18 & AF & Normal & Normal \\
& 21 & Brain & Normal & Normal \\
& 21 & Heart & Normal & Normal \\
2 UPD15mat & 21 & Skin & Normal & Normal \\
& 14 & AF & PWS & PWS \\
& 15 & Tongue & PWS & PWS \\
& 15 & Stomach & PWS & PWS \\
& 15 & Skin & PWS & PWS \\
3 UPD15mat & Cord & PWS & PWS \\
& 15 & AF & PWS & PWS \\
& 16 & CVS & PWS & Hypomethylation \\
& 20 & Placenta & PWS & Pypomethylation \\
& 20 & Eye & PWS & PWS \\
& 20 & Oesophagus & PWS & PWS \\
& 20 & Intestine & PWS & PWS \\
& 20 & Lung & PWS & PWS \\
& 20 & Liver & PWS & PWS \\
& 20 & Kidney & PWS & PWS \\
& 20 & Skeletal & PWS & PWS \\
& 20 & Muscle & PWS & PWS \\
& 20 & Skin & PWS & PWS \\
\hline
\end{tabular}

$\mathrm{AF}=$ amniotic fluid cells, $\mathrm{CVS}=$ chorionic villus samples. sites in the $\mathrm{CpG}$ island of the SNRPN gene. Also, a $6.6 \mathrm{~kb}$ undigested band was seen in LB samples with a maternal deletion (fig 1 , bottom panel, lanes 5 and 6), suggesting that the $C f o I$ sites at PW71 are hypermethylated on the paternal chromosome 15 compared with the methylation status at the NotI sites in the CpG island of the SNRPN gene.

\section{ANALYSIS OF FETAL TISSUES}

Examples of SNRPN methylation in AF, CVS, and fetal tissue samples are shown in fig 2 . Like the LB cell lines, the SNRPN methylation results were consistent with those of DNA polymorphism analysis for DNA isolated directly from fetal tissues and after cell culture. Results of the methylation assay in fetal tissues showed complete consistency with those of the corresponding AF samples (table 2). Conversely, the PW71 methylation assay showed multiple smaller bands in CVS and placental tissue of case 3 which was not consistent with DNA polymorphism analysis (table 2), suggesting hypomethylation at the $C f o I$ sites in these tissues.

\section{Discussion}

The diagnostic utility of differential methylation at PW71 and in the $\mathrm{CpG}$ island of the SNRPN gene has been shown in a large patient series using peripheral blood samples. ${ }^{78}$ However, other tissues have not been extensively examined to validate their usefulness for diagnosis of PWS/AS. Therefore, we performed an extensive analysis of the parent of origin specific methylation in LB cell lines, cultured AF samples, cultured CVS, and various fetal tissues. We have confirmed that DNA methylation in the CpG island of the SNRPN gene is consistent with other molecular cytogenetic tests in all of these tissues, whereas unstable or inconsistent methylation patterns were found at PW71 in many of these tissue samples.

Since results of the SNRPN methylation were completely consistent with results by DNA polymorphism or FISH in LB cell lines analysed, these cell lines provide an alternative source of material for the SNRPN methylation 
assay. However, we found discrepancies between results of the PW71 methylation assay and previous results of DNA polymorphism or FISH in $17 \%$ of LB cell lines. In our study, some LB cell lines showed either hypermethylation or hypomethylation at the $C f o \mathrm{I}$ sites at the PW71 site.

We also found hypomethylation in CVS and one placental tissue using the PW71B probe. These results were consistent with previous reports where hypomethylation has been shown at PW71 in CVS, ${ }^{910}$ placenta, and several tumours ${ }^{10}$ although a consistent methylation pattern has been shown in a few AF samples. ${ }^{910}$

Appropriate SNRPN methylation patterns have recently been reported in several fetal tissues with the exception of fetal testes. ${ }^{16}$ In our study, cultured cells from AF and CVS showed concordant results between the SNRPN methylation and DNA polymorphism analysis. Results of the SNRPN methylation assay were also consistent between AF, CVS, and fetal tissues. These results suggest that SNRPN methylation in either cultured AF cells or cultured CVS cells may accurately reflect the methylation status of the fetus.

Recently several familial PWS/AS families have been reported in which small deletions were identified at the region between the PW71 locus and the SNRPN gene which could not be detected using either commercially available FISH probes or DNA polymorphism analysis with current markers. These deletions are thought to encompass a putative imprinting centre $^{12}$ and affected subjects in these families show abnormal methylation patterns. Recurrence risk in these families is high $(50 \%)$ for carriers of the imprinting mutation. For these cases, the SNRPN methylation analysis could provide a prenatal diagnosis option using AF samples or CVS.

\section{Conclusion}

The data presented here confirm that the SNRPN methylation assay is consistent with other molecular cytogenetic tests in all tissues examined. Our data verify the usefulness of the SNRPN methylation assay in diagnosis of PWS/AS using LB cell lines, cultured AF samples, and CVS, which can, for the first time, provide prenatal diagnosis for families with imprinting mutations.

The authors wish to thank Drs James S Sutcliffe and Arthur L Beaudet for providing the SNRPN exon $\alpha$ DNA probe and Dr Bernhard Horsthemke for the PW71B probe. We also greatly appreciate the efforts of Dr Susan H Black, Dr Anne Maddalena, and Ms Carol Ording (Genetics \& IVF Institute, VA) for providing control CVS samples. This study was supported in part by the Beneficia Foundation (DHL).

1 Sutcliffe JS, Nakao M, Christian S, et al. Deletions of a differentially methylated $\mathrm{CpG}$ island at the SNRPN gene define a putative imprinting control region. Nat Genet 994;8:52-8.

2 Buiting K, Saitoh S, Gross S, et al. Inherited microdeletions in the Angelman and Prader-Willi syndromes define an imprinting centre on human chromosome 15. Nat Genet 1995;9:395-400.

3 Kuwano A, Mutirangura A, Dittrich B, et al. Molecular dissection of the Prader-Willi/Angelman syndrome region (15q11-13) by YAC cloning and FISH analysis. Hum Mol Genet 1992;1:417-25.

4 Teshima I, Chadwick D, Chitayat $\mathrm{D}$, et al. FISH detection of chromosome 15 deletions in Prader-Willi and Angelman syndromes. Am 7 Med Genet 1996;62:216-23.

5 Mutirangura A, Greenberg F, Butler MG, et al. Multiplex PCR of three dinucleotide repeats in the Prader-Willi Angelman critical region (15q11-q13): molecular diagAngelman critical region (15q11-q13): molecular diagnosis and mechanism

6 Smith A, Prasad M, Deng ZM, Robson L, Woodage T, Trent RJ. Comparison of high resolution cytogenetics, fluorescence in situ hybridization, and DNA studies to validate the diagnosis of Prader-Willi and Angelman's syndromes. Arch Dis Child 1995;72:397-402.

7 Gillessen-Kaesbach G, Gross S, Kaya-Westerloh S, Passarge E, Horsthemke B. DNA methylation based testing of 450 patients suspected of having Prader-Willi syndrome. $f \mathrm{Med}$ Genet 1995;32:88-92.

8 Kubota T, Sutcliffe JS, Aradhya S, et al. Validation studies of SNRPN methylation as a diagnostic test for PraderWilli syndrome. Am $₹$ Med Genet (in press).

9 Van den Ouweland AMW, Van der Est MN, Wesby-van Swaay E, et al. DNA diagnosis of Prader-Willi and Angelman syndromes with the probe PW71 (D15S63). Hum Genet 1995;95:562-7.

10 Dittrich B, Buiting K, Gross S, Horsthemke B. Characterization of a methylation imprint in the Prader-Willi syndrome chromosome region. Hum Mol Genet 1993;2: 1995-9.

11 American Society of Human Genetics/American College of Medical Genetics Test and Technology Transfer Committee. Diagnostic testing for Prader-Willi and Angelman syndromes: report of the ASHG/ACMG test and technology transfer committee. Am $\mathcal{F}$ Hum Genet 1996;58: nology tran

12 Surh LS, Wang H, Hunter AGW. Deletion and uniparental disomy involving the same maternal chromosome $15 . N$ Engl f Med 1994;330:572-3.

13 Christian SL, Smith ACM, Macha M, et al. Prenatal diagnosis of uniparental disomy 15 following trisomy $15 \mathrm{mo}$ saicism. Prenat Diagn 1996;16:323-32.

14 Dittrich B, Robinson WP, Knoblauch H, et al. Molecular diagnosis of the Prader-Willi and Angelman syndromes by detection of parent-of-origin specific DNA methylation in 15q11-13. Hum Genet 1992;90:313-15.

15 Glenn CC, Nicholls RD, Robinson WP, et al. Modification of 15q11-q13 DNA methylation imprints in unique Angelman and Prader-Willi patients. Hum Mol Genet 1993;3: 1377-82.

16 Glenn CC, Saitoh S, Jong MTC, et al. Gene structure, DNA methylation, and imprinted expression of the human SNRPN gene. Am ₹ Hum Genet 1996;58:335-46. 